\title{
Potts Glass on Random Graphs
}

\author{
'Florent KrząKąA ${ }^{1}$ and LenKa Zdeborová ${ }^{23}$ \\ 1. PCT, UMR 7083 CNRS-ESPCI, 10 rue Vauquelin, 75231 Paris, France \\ 2 Université Paris-Sud, LPTMS, UMR8626, Bât. 100, Université Paris-Sud 91405 Orsay cedex, France \\ CNRS, LPTMS, UMR8626, Bât. 100, Université Paris-Sud 91405 Orsay cedex, France
}

PACS 75.10.Nr - Spin-glass and other random models

PACS 89.20.Ff - Computer science and technology

\begin{abstract}
We solve the $q$-state Potts model with anti-ferromagnetic interactions on large random lattices of finite coordination. Due to the frustration induced by the large loops and to the local tree-like structure of the lattice this model behaves as a mean field spin glass. We use the cavity method to compute the temperature-coordination phase diagram and to determine the location of the dynamic and static glass transitions, and of the Gardner instability. We show that for $q \geq 4$ the model possesses a phenomenology similar to the one observed in structural glasses. We also illustrate the links between the positive and the zero-temperature cavity approaches, and discuss the consequences for the coloring of random graphs. In particular we argue that in the colorable region the one-step replica symmetry breaking solution is stable towards more steps of replica symmetry breaking.
\end{abstract}

There are mainly two types of lattices for which the mean field theory of spin glasses is exact. The first type is the fully connected lattice underlying the canonical Sherrington-Kirkpatrick model [1]. Parisi introduced the replica symmetry breaking (RSB) scheme [2] to obtain a solution of this model that was much later proven to be rigorously exact [3]. Similar solutions have been derived - for the p-spin model [4] and for the Potts glass model [5], and these played a major role in the development of the mean field theory of the structural glass transition $[6,7]$.

The second type of lattice for which the mean field theory is exact is given by large random graph with fixed average degree (or connectivity), a case commonly refereed to as "Bethe lattice" in the physics literature [8]. These lattices are considered as more realistic since the notions of distance and neighboring can be naturally defined. Few years ago, Mézard and Parisi $[9,10]$, using the cavity method, have adapted the replica symmetry breaking scheme to solve models on such sparse lattices. The theoretical activity following this breakthrough was mainly concentrated on the zero temperature limit [10] of various models on random graphs, motivated by the equivalence with the random cases of hard combinatorial optimization problems such as graphs coloring or the satisfiability of boolean formulae [11]. The last few years have indeed witnessed spectacular successes in this direction [11].

In this paper, we consider a $q$-state Potts model with anti-ferromagnetic nearest neighbors interactions on random graphs. Due to the frustration induced by large loops and to the locally tree-like structure of the graph, the model actually behaves as a mean field spin glass. Using the cavity approach, we study systematically the phase diagram for different temperatures $T$, average connectivities $c$, and number of states (colors) $q$. Determining the different phases, and which step of replica symmetry breaking is needed for given $T, c, q$ are the main contributions of this work. The zero temperature limit of this model maps to the $q$-coloring problem, and has been studied extensively [12-18], while the positive temperature studies are limited to $[13,16,18]$. Our results give a generic picture of Potts glasses on sparse random graphs, and have interesting consequences for constraint satisfaction problems as well. Finally, we also discuss the subtle connections between the positive [9] and zero temperature [10] cavity formalisms, and correct some confusions that have been made in the literature in the use of these two approaches.

Anti-ferromagnetic Potts glass. - We consider a $q$-state anti-ferromagnetic Potts model. Given a graph $G=(\mathcal{V}, \mathcal{E})$ defined by its vertices $\mathcal{V}=\{1, \ldots, N\}$ and edges $(i, j) \in \mathcal{E}$ the Hamiltonian reads

$$
\mathcal{H}=\sum_{(i, j) \in \mathcal{E}} \delta\left(\sigma_{i}, \sigma_{j}\right)
$$


where $\sigma=1, \ldots, q$ are the values of the different Potts spins. On a bipartite graph (like a $d$-dimensional lattice), the ground-state is anti-ferromagnetically ordered. On large random graphs, however, the frustration induced by the large loops does not allow the anti-ferromagnetic order and a glassy solution appears instead.

We will consider two ensembles of sparse random graphs: the Erdös-Rényi (ER) graphs with Poissonian degree distribution of mean $c$, and the regular graphs with fixed degree $c$. Both are locally tree-like in the thermodynamic limit so that the length of the shortest loop going through a random vertex diverges as $\log N$ when $N \rightarrow \infty$ (our results generalize to other locally tree-like ensembles).

It is interesting to discuss the relation of the model defined by the Hamiltonian (1) with the one defined by the following disordered Hamiltonian

$$
\hat{\mathcal{H}}=\sum_{i<j:(i, j) \in \mathcal{E}} \delta\left(\sigma_{i}, \pi_{i j}\left(\sigma_{j}\right)\right)
$$

where $\pi_{i j}$ represents a random permutation of the $q$ colors. In this disordered anti-ferromagnetic Potts model, the anti-ferromagnetic order is explicitly destroyed by the disorder independently of the lattice. Notice that a different disordered Potts model was defined originally [5, 19] but the advantage of our definition (2) is that due to the existence of a gauge symmetry, the total equilibrium magnetization is zero at all temperatures, independently of the lattice. Indeed, if we change the color of an arbitrary spin $i$, then there always exists an energy conserving gauge transformation of the interactions between spin $i$ and its neighbors such that the probability of the new set of interactions is the same as of the original one. After averaging over disorder the magnetization is zero, and self-averaging implies that it is also zero on a single large system. The model defined by (2) is thus convenient for numerical simulations of the glassy phase, even on regular finite dimensional lattices. Note that a ferromagnetic version of (2) was already defined and studied in [20].

The two models defined by (11) and (2) share the same solution on sparse random lattices. Indeed, by the gauge transformation, the two models can be made equivalent on a large tree-like neighborhood of a vertex, the difference being pushed on the boundary of this neighborhood. Inside a pure state the boundary conditions caused by the rest of the graph are uncorrelated and color symmetric (a color asymmetric, ordered, solution is ruled out by the frustrating long loops), thus a point-wise random permutation does not change their statistical properties and the same solution is obtained for both the models. We actually use this equivalence to resolve the numerical instability towards the anti-ferromagnetic solution in the cavity iterative equations for the model (11) by randomly permuting the colors after each iteration, which is exactly what the Hamiltonian (2) would do.

Cavity methodology reminder. - The cavity method [9] allows to solve models (112) on large sparse random graphs. The results are expected to be exact (although a rigorous proof is still missing) provided that the correct level of replica symmetry breaking is considered. A detailed derivation of the equations for Hamiltonian (1) is presented in [18], we discuss only the parts necessary for the presentation of our results.

The liquid phase. The high-temperature liquid, or paramagnetic, phase is characterized by an exponential decay of correlation functions and is associated with the existence of a single pure state. The simplest version of the cavity method, called replica symmetric (RS), describes correctly this phase $[13,18]$. A first, simple, way to check the appearance of a spin glass phase is to compute the temperature where the spin-glass susceptibility diverges, or equivalently where the RS cavity equations do not converge on a single graph or, in other words, where the RS solution is locally unstable. When this happens, a continuous phase transition towards a spin glass phase arises. The temperature of the local RS instability is given by [18]

$$
T_{\text {local }}(q, c)=-1 / \ln \left(1-\frac{q}{\sqrt{\kappa}+1}\right),
$$

where $\kappa=c$ for ER graph, and $c-1$ for regular graphs.

It is however common to observe the discontinuous appearance of a spin glass solution before the local instability sets in. In order to check this point, it has been argued recently $[16,21]$ that the decay of the so-called point-to-set correlations provides a necessary and sufficient condition for the validity of the RS solution. When the temperature is lowered, a dynamical temperature $T_{d}$ might be reached below which the point-to-set correlation does not decay to zero and the system undergoes a dynamical phase transition, as the equilibration time of "local flip" Monte-Carlo dynamics diverges [21]. As shown in [16], this correlation does not decay to zero if and only if it exists a non-trivial solution to the one-step replica symmetry breaking equation when the so-called Parisi parameter $m=1$ (see below). Below $T_{d}$ the RS pure state splits into exponentially many disconnected pure states.

The glass phase with many states. The one-step replica symmetry breaking (1RSB) cavity method deals with this situation. It allows to compute the complexity $\Sigma(f)$ defined as the logarithm of the number of pure states of internal free energy density $f$. In order to do so, we follow [22] and define a potential $\Phi(\beta, m)$ as

$$
e^{-\beta m N \Phi(\beta, m)}=\sum_{\alpha} e^{-\beta m N f_{\alpha}(\beta)}=e^{N[-\beta m f(\beta)+\Sigma(f)]},
$$

where the sum is over all states $\alpha$, and $m=\partial_{f} \Sigma(f) / \beta$ a temperature-like parameter in the Legendre transform $[9$, $22]$. In the closely related replica formalism, the parameter $m$ defines the block size of Parisi's overlap matrix [2].

For regular graphs $\Phi(\beta, m)$ can be computed from the 


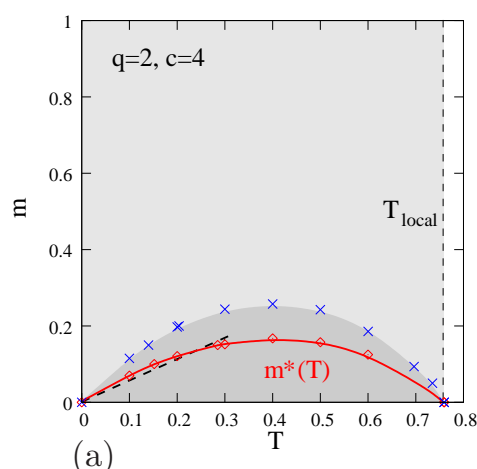

(a)

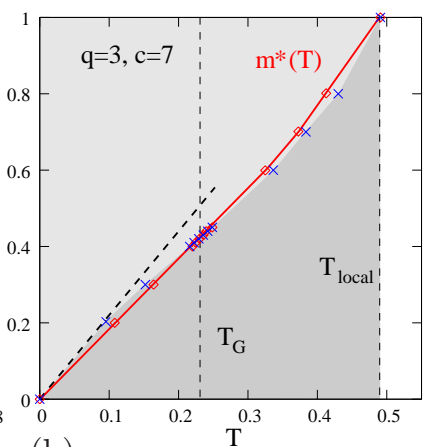

(b)

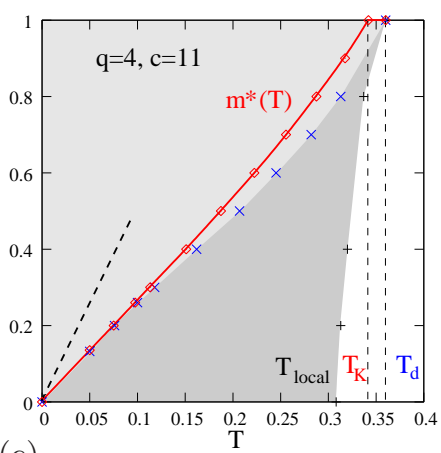

(c)

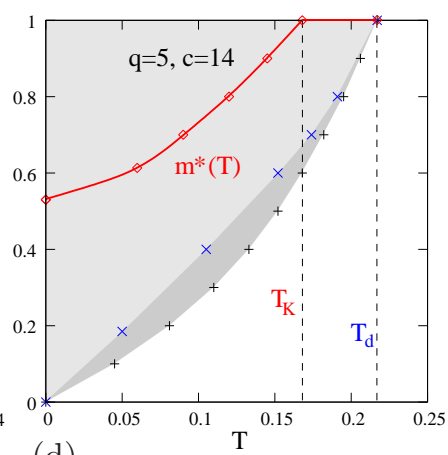

(d)

Fig. 1: A set of m-T diagrams for the $q$-state anti-ferromagnetic Potts model on $c$-regular random graphs: a non-trivial solution of eq. (5) exists in the shaded region. The curves with diamond data points $m^{*}(T)$ (red online) represent the thermodynamic value of the parameter $m$. The darker region, delimited with crosses (blue online) is type II unstable towards further steps of replica symmetry breaking. The dotted lines (absent in (d)) represent the type I instability $m_{I}(T)$ calculated analytically close to $T=0,1 \mathrm{RSB}$ is type I unstable for $m>m_{I}(T) . T_{d}, T_{K}, T_{G}$ and $T_{\text {local }}$ are the dynamical, Kauzmann, Gardner and local temperature (respectively). (a) is an example of continuous (Sherrington-Kirkpatrick-like) transition towards more than one step of RSB; (b) is an example of continuous transition to the 1RSB phase, with a Gardner transition at lower temperatures; (c) is a discontinuous transition in the uncolorable phase and (d) is a discontinuous transition in the colorable phase.

fixed point of the $1 \mathrm{RSB}$ equation

$$
P(\vec{\psi})=\frac{1}{Z_{1}} \prod_{k=1}^{c-1} \int d \vec{\psi}^{k} P\left(\vec{\psi}^{k}\right) \delta\left[\vec{\psi}-\overrightarrow{\mathcal{F}}\left(\left\{\vec{\psi}^{k}\right\}\right)\right]\left(Z_{0}\right)^{m}
$$

where $P(\vec{\psi})$ is the unknown probability distribution of $q$ component normalized vectors $\vec{\psi}, Z_{1}$ a normalization, and the function $\mathcal{F}$ is defined component-wise as

$$
\psi_{r}=\mathcal{F}_{r}\left(\left\{\psi_{r}^{k}\right\}\right)=\frac{1}{Z_{0}} \prod_{k=1}^{c-1}\left[1-\left(1-e^{-\beta}\right) \psi_{r}^{k}\right],
$$

where $r=1 \ldots q, Z_{0}$ is a normalization constant and each component of the vector $\vec{\psi}$ is the probability that a node, in absence of one of its neighbors, takes the corresponding color [9]. For graphs with fluctuating degree eq. (5) has to be written edge-dependently and an average over the edges of the graph has to be performed $[9,18]$. In both cases, eq. (5) can be solved numerically using the population dynamics method [9] where the probability distribution $P(\vec{\psi})$ is represented by a population of many elements $\vec{\psi}$. At $m=1$, a formal substitution introduced in [16] (see also [18]) allows to rewrite the averaging of eq. (5) in a simple way for the fluctuating connectivity graphs as well.

The two-temperatures diagram. - The temperature $T$ (resp. temperature-like parameter $m$ ) in (5) is related to the energy (resp. size) of the states. It is thus convenient to consider the solution of eq. (5) in the $m-T$ diagram. This representation moreover clarifies the connection of the positive temperature approach with the different zero-temperature limits of $[12,14]$ and $[15,18]$. We plot several such diagrams in fig. 1. The region where a non-trivial (different from the RS one) solution of (5) exists is shaded in light and darker grey. At $m=1$ a non-trivial solutions exists for all $T \leq T_{d}$. At $m=0$ the absence of the re-weighting term $Z_{0}^{m}$ makes the solution of (5) be non-trivial if the RS equations do not converge on a single graph, i.e., for all $T<T_{\text {local }}$.

The next step is the computation of the thermodynamic value of the parameter $m^{*}(T)$. The free energy in the $1 \mathrm{RSB}$ phase reads

$$
-\beta f_{1 \mathrm{RSB}}=\max _{f: \Sigma(f) \geq 0}[-\beta f+\Sigma(f)] .
$$

Call $\tilde{f}$ the free energy for which $\partial_{f} \Sigma(\tilde{f})=\beta$ (so that $m=$ 1 , see eq. (4) $)$. If $\Sigma(\tilde{f}) \geq 0$ then $-\beta f_{1 \mathrm{RSB}}=-\beta \tilde{f}+\Sigma(\tilde{f})=$ $-\beta \Phi(\beta, 1)=-\beta f_{\mathrm{RS}}$ and the free energy is equal to the RS one. This explains the importance of the value $m=1$ : as long as the complexity $\Sigma(m=1)>0$, then $m^{*}=1$. If $\Sigma(m=1)<0$, however, the pure states corresponding to the free energy $\tilde{f}$ are almost surely absent in the large size limit and to maximize eq. (17) we choose instead $f^{*}$ such that $\Sigma\left(f^{*}\right)=0$. Consequently $f_{1 \mathrm{RSB}}=f^{*}>f_{\mathrm{RS}}$ and a genuine phase transition happens at the so-called Kauzmann temperature $T_{K}$ where $\Sigma(m=1)=0$.

Between $T_{d}$ and $T_{K}$ the thermodynamics is dominated by an exponential number of states and the total free energy is equal to liquid one, so that the dynamical transition is not a true transition in the Ehrenfest sense. Below the Kauzmann temperature the thermodynamics is dominated only by a finite number of them (although exponentially many states still exist, they are thermodynamically negligible). At $T=T_{K}$, the free energy has a discontinuity in its second derivative, i.e., in the specific heat. This phenomenon, first hypothesized by Kauzmann, is called the ideal glass transition.

Zero temperature limit. The q-state antiferromagnetic Potts model maps to a fundamental problem of graph theory: if the ground state energy 
is zero, the graph is colorable by $q$ colors so that neighboring spins have different colors, otherwise the graph is $q$-uncolorable. This has been studied in great detail in [12-18]. An important relation is that if $m^{*}(T=0)>0$, then the ground state energy is zero and the graph is $q$-colorable. If $m^{*}(T=0)=0$, however, the ground state energy is positive and the curve $m^{*}(T)$ approaches the origin with a tangent $y=m / T$.

The limit $T \rightarrow 0$ at fixed ratio $m / T$ corresponds to the original zero-temperature solution of the coloring problem $[12,14]$ and more generally to the so-called "energetic" cavity method introduced in [10]. In the $m-T$ diagrams, all curves $m(T)$ starting at the origin $(0,0)$ have therefore their counterpart in $y=\mathrm{d} m(T) /\left.\mathrm{d}(1 / T)\right|_{T=0}$ in the zero temperature formalism. It is actually a non-trivial check of our numerical results that the different curves $m(T)$ we found match perfectly the corresponding slopes $y$ computed in $[12,14]$. This shows however that the zero temperature "energetic" approach [10-12] does not describe correctly the thermodynamical states in the colorable phase (where $m^{*}>0$ ). The results of this approach should thus be used with caution, and have a clear meaning only in the $m-T$ phase diagram. This warning applies in particular to the critical values for the dynamical and the $1 \mathrm{RSB}$ stability transitions in $[7,11,12,14,23]$. A complementary "entropic" zero temperature approach was however introduced in [15] and allows to obtain more informations about the zero energy states $[17,18]$.

Gardner instability. In the mean field spin glass theory, the 1RSB description may be wrong at low temperatures, in which case more steps of RSB are necessary: this phenomena is called the Gardner transition [5, 24]. To test the validity of the 1RSB, it is widely considered as sufficient to perform a local stability analysis of the 1RSB approach $[7,14,23,25]$. Two types of perturbation are to be considered. In the first type, one checks if the 1RSB iterations (5) are stable towards small changes in the site dependent distributions $P(\vec{\psi})$. In the second type, one checks if the 1RSB iterations (5D) are stable against small changes in the probabilities $\vec{\psi}$ themselves.

The instability of the first type at positive temperature is quite problematic to be checked. We used the analytical results of [14] for the $m, T \rightarrow 0$ limit with $y=m / T$ fixed (see the dotted lines $m_{I}$ in fig. 11). It is moreover a simple algebraic fact that the averages of the 1RSB distributions $P(\vec{\psi})$ at $m=1$ follow the RS equations [17,18]: for $m=1$ the 1RSB approach is thus type-I unstable for $T<T_{\text {local }}$. We conjecture that the contrary is also true, i.e. that non-convergence of the distribution $P(\vec{\psi})$ at $m=1$ would manifest itself as a non-convergence of its mean.

The instability of the second kind can also be studied analytically in the $m, T \rightarrow 0$ limit [14], and for generic $(m, T)$ numerically as follows: one first find the fixed point of eq. (5) using the population dynamics method, and then compute how small differences in the populations evolve in the iteration. This can be done by creating a second copy of the population representing the distribution $P(\vec{\psi})$, perturbing infinitesimally every of its elements and checking if the two populations converged to the same one for long times, or equivalently by using the more involved numerical method developed in [25], which we also used to cross-check our results.

We observed that, at a given temperature, the 1RSB states corresponding to $m>m_{I}$ are type I unstable while those corresponding to $m<m_{I I}$ are type II unstable. In order to check the stability, one thus needs to compare $m_{I}(T)$ and $m_{I I}(T)$ with the thermodynamical $m^{*}(T)$. Generically, the instability of the second type is the relevant one. Some examples are shown in fig. 1 in (a) for $q=2, c=4$ the whole glassy phase is 1RSB unstable. In (b), $q=3, c=7$, the 1 RSB solution is type II unstable for $T<T_{G}$ and stable for $T>T_{G}$. We cannot rule out a type I instability, yet we believe this instability unlikely, given the slope of $m_{I}(T)$ at the origin. We also implemented the 2RSB equations and were not able to find any non-trivial solutions in the type II stable region. For (c) $q=4, c=11$ and (d) $q=5, c=14$ we observed that the whole low temperature phase is $1 \mathrm{RSB}$ stable.

Overlap distributions. The overlap probability distribution function is a useful order parameter in spin glasses [2] and allows to characterize the different phases. Consider two configurations $\alpha$ and $\beta$ chosen uniformly at random at given temperature. To define their overlap we use the matrix $Q^{\mu \nu}=\sum_{i=1}^{N} \delta\left(\sigma_{i}^{\alpha}, \mu\right) \delta\left(\sigma_{i}^{\beta}, \nu\right) / N$ and an example of the definition of an overlap which is invariant under permutation of colors in one of the configurations is $Q=\sum_{\mu, \nu=1}^{q} Q^{\mu \nu}$. In the liquid phase, the equilibrium distribution of the overlap $P(Q)$ is just a delta function. Below the dynamic transition, it is the same, and becomes non-trivial only for $T<T_{K}$. In this case, the $P(Q)$ is made of two peaks, their relative weights are a monotonous function of the parameter $m^{*}$. For $m^{*} \rightarrow 1$ the weight of the second peak is near to zero, for $m^{*} \rightarrow 0$ there is only one dominating state and the weight of the second peak is near to one. For $T<T_{G}$ the distribution $P(Q)$ is continuous as in the fully connected Ising spin glass model [2]. At zero temperature the distribution $P(Q)$ is nontrivial only if $0<m^{*}(T=0)<1$ (i.e. in the colorable phase. 1)

The phase diagram. - We now present our results and summarize our findings for the temperatureconnectivity phase diagrams. We computed the dynamical $T_{d}$ and Kauzmann $T_{K}$ temperatures both for regular and Erdős-Rényi graphs via the simplification of eq. (5) at $m=1$ [16]. The Gardner temperature $T_{G}$ is computed only in the simpler case of regular graphs.

Ising case: $q=2$. For the Ising spins, the spin glass transition arises continuously at $T_{\text {local }}=T_{d}=T_{K}$ given by eq. (3). We found that the thermodynamic states given by $m^{*}(T)$ are always 1RSB unstable as $m^{*}<m_{I I}$ (see

\footnotetext{
${ }^{1}$ The triviality of $P(Q)$ at zero temperature was predicted in [26] but the argumentation in [26] is valid only in the uncolorable phase.
} 
Potts Glass on Random Graphs
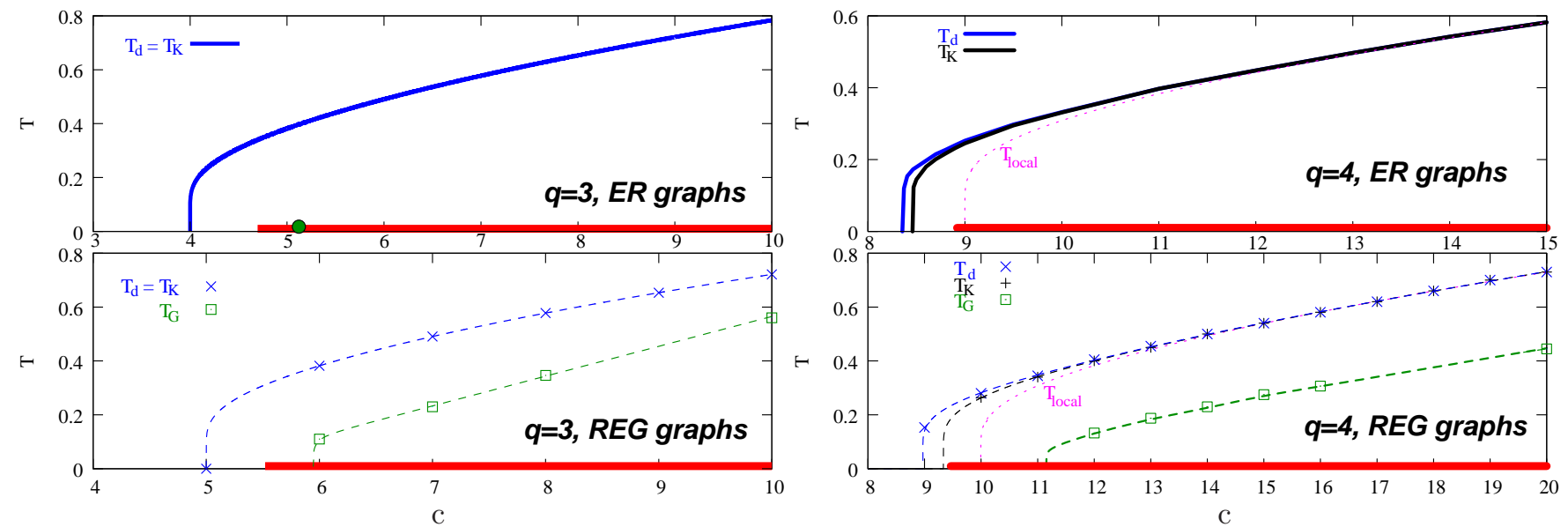

Fig. 2: Phase diagrams for the 3-state (left) and 4-state (right) anti-ferromagnetic Potts glass on Erdős-Rényi graphs of average degree $c$ (top) and regular graphs of degree c (bottom). For $q=3$ the transition is continuous $T_{d}=T_{K}=T_{\text {local }}$. For $q=4$, we find that $T_{d}>T_{K}>T_{\text {local }}$, while for larger connectivities these three temperatures become equal. The Gardner temperature $T_{G}$ for regular graphs is also shown (green online). In the case of $q=3$ and ER graphs, $T_{G}>0$ only for connectivities larger than $c>c_{G}=5.08$ [14]. The bold (red online) lines at zero temperature represent the uncolorable connectivities $c>c_{s}$.

the example in fig. 1(a)). Ising spin glasses should thus be described via the infinite replica symmetry breaking formalism as pointed out already in [9]. We also notice that both the curves $m^{*}(T), m_{I I}(T)$ both goes to zero when $T \rightarrow T_{\text {local }}^{-}$, as in the fully connected model. In the zero temperature limit the random graphs are 2-uncolorable in the spin glass phase, i.e., when $T_{\text {local }}>0$ (for $c>1$ on ER graphs and $c>2$ on regular graphs [13]).

The case $q=3$. The phase diagrams are shown in fig. 2 (left). We again observe, for both ER and regular graph, a continuous transition at $T_{\text {local }}=T_{d}=T_{K}$ given by eq. (3). However, the situation is different from the Ising case. Consider for instance the case $c=7, q=3$ in fig. 1(b): first, $m^{*}\left(T_{\text {local }}\right)=1$ and $m_{I I}\left(T_{\text {local }}\right)=1$, and moreover the thermodynamical states are type II stable for $T>T_{G}$. Even if we have not ruled out completely the instability of type I, this makes plausible the presence of a continuous transition towards a 1RSB stable phase, just like in the fully connected 3-state Potts model of [5].

We found that the Gardner temperature is positive only in the uncolorable phase. See table 1 for critical values in regular graphs. For ER graphs it was already shown that $T_{G}>0$ only for $c>5.08$ [14]. The zero temperature study [14] also indicates that both instabilities are irrelevant close to the colorable threshold. All this suggests that, for colorable connectivities, the whole low temperature phase of the 3 -state Potts model is 1RSB stable.

The case $q \geq 4$. In this generic case we observe the same set of transitions as in the mean field theory for the ideal glass transition in structural glasses. We plot the $q=4$ phase diagram in the right panel of fig. 22 The discontinuous dynamical transition $T_{d}$ first arises followed by the Kauzmann transition at $T_{K}<T_{d}$. The Gardner transition $T_{G}<T_{K}$ arises again for connectivities larger

\begin{tabular}{|c|c|c|c|c|c|c|}
\hline q & $c$ & $T_{\mathrm{d}}$ & $T_{\mathrm{K}}$ & $T_{\text {local }}$ & $T_{\mathrm{G}}$ & $\mathrm{col}$ \\
\hline$\overline{2}$ & 3 & \multicolumn{4}{|c|}{0.567} & no \\
\hline 2 & 4 & \multicolumn{4}{|c|}{0.759} & no \\
\hline 3 & 5 & \multicolumn{3}{|c|}{0} & $\mathrm{xxx}$ & yes \\
\hline 3 & 6 & \multicolumn{3}{|c|}{0.381} & $0.11(1)$ & no \\
\hline 3 & 7 & \multicolumn{3}{|c|}{0.490} & $0.23(1)$ & no \\
\hline 3 & 8 & \multicolumn{3}{|c|}{0.577} & $0.34(2)$ & no \\
\hline 3 & 10 & \multicolumn{3}{|c|}{0.721} & $0.56(3)$ & no \\
\hline 4 & 9 & $0.153(5)$ & $\mathrm{xxx}$ & $\mathrm{xxx}$ & $\mathrm{xxx}$ & yes \\
\hline 4 & 10 & $0.280(5)$ & $0.264(5)$ & 0 & $\mathrm{xxx}$ & no \\
\hline 4 & 11 & $0.345(5)$ & $0.340(5)$ & 0.308 & $\mathrm{xxx}$ & no \\
\hline 4 & 12 & $0.405(5)$ & $0.400(5)$ & 0.382 & $0.135(5)$ & no \\
\hline 4 & 13 & $0.455(5)$ & $0.450(5)$ & 0.441 & $0.185(5)$ & no \\
\hline 4 & 14 & $0.500(5)$ & $0.500(5)$ & 0.492 & $0.230(5)$ & no \\
\hline 4 & 15 & $0.540(5)$ & $0.540(5)$ & 0.539 & $0.275(5)$ & no \\
\hline 4 & 16 & $0.581(5)$ & $0.581(5)$ & 0.581 & $0.305(5)$ & no \\
\hline 4 & 20 & $0.730(5)$ & $0.730(5)$ & 0.730 & $0.445(5)$ & no \\
\hline 5 & 14 & $0.214(5)$ & $0.168(5)$ & $\mathrm{xxx}$ & $\mathrm{xxx}$ & yes \\
\hline 5 & 15 & $0.276(5)$ & $0.255(5)$ & $\mathrm{xxx}$ & $\mathrm{xxx}$ & no \\
\hline 5 & 16 & $0.322(5)$ & $0.306(5)$ & $\mathrm{xxx}$ & $\mathrm{xxx}$ & no \\
\hline 5 & 17 & $0.360(5)$ & $0.348(5)$ & 0 & $\mathrm{xxx}$ & no \\
\hline 5 & 18 & $0.396(5)$ & $0.386(5)$ & 0.268 & $0.09(2)$ & no \\
\hline 5 & 19 & $0.428(5)$ & $0.420(5)$ & 0.325 & $0.13(1)$ & no \\
\hline 5 & 20 & $0.460(5)$ & $0.452(5)$ & 0.369 & $0.16(1)$ & no \\
\hline
\end{tabular}

Table 1: Critical temperatures $T_{d}, T_{K}, T_{\text {local }}$ and $T_{G}$ for the regular graphs ensemble and the $q$-colorability of the graphs. The error bars come from the numerical precision in evaluation the solution of (5) by the population dynamics method.

than the colorable thresholds. It is also interesting to notice that as the connectivity $c$ grows, one actually observes that $T_{d}, T_{K} \rightarrow T_{\text {local }}$ (see table 1) so that a situation similar to $q=3$ is recovered. This happens however for con- 
nectivities well beyond the coloring transition $c_{s}$ (since $c_{s} \propto 2 q \log q$ while $c_{\text {local }} \propto q^{2}$.).

Our results on the 1RSB type II stability, and the conjecture that the system is type I stable for $T>T_{\text {local }}$ at all $m \leq 1$, have important implications in the $q$-coloring problem: for $q \geq 4$ the colorable phase is always 1RSB stable. The expert reader will notice that the conclusions in [14] were different and that a unstable region was found, [14] however describes the region $m, T \rightarrow 0$, which is not relevant for the thermodynamical states in the colorable phase. While most clusters might be instable, the relevant ones are always stable in the colorable phase.

Discussion. - We studied in this paper a mean field Potts glass with anti-ferromagnetic (or disordered) interactions. We observed a continuous glass transition for $q=2,3$ and a discontinuous one for $q \geq 4$ (at least for low enough connectivities). We also considered the stability of the 1RSB solution and concluded, in particular, that the colorable phase at $T=0$ is stable for $q \geq 4$ and probably for $q=3$ as well, thus correcting previous claims [14]. We expect this conclusion to be also valid for the satisfiability problem [23]. It is, however, important to develop a way to confirm properly the type I stability for $q=3$.

The phase diagram for $q \geq 4$ (right panel of fig. 2) has many common characteristics with the one of the finite connectivity p-spin model [25,27], whose zero temperature limit maps to the XOR-SAT problem [28]. Let us, however, state several differences: The satisfiability threshold $c_{s}$ corresponds to the Kauzmann one $c_{K}$ in [25,27], while they are different in our model. The temperatures $T_{K}$ and $T_{d}$ are different in the large connectivity limit in $[25,27]$ where no local instability exists 2 , while $T_{K}$ and $T_{d}$ converge to the same value $T_{\text {local }}>0$ for large connectivities in our model. This illustrates the differences (and the richness) in the phenomenology of the coloring and satisfiability problems when compared to XOR-SAT.

Our model is also very similar to the lattice glass model [7] where the inverse temperature is replaced by the chemical potential $\mu$ and the energy by the density of particles $\rho$. The close packing limit studied in [7] however concerns only the $m=0, \mu=\infty$ limit. A quantitative study of the phase diagram with the correct value for the dynamical, Kauzmann and Gardner transition in this model is thus still missing and we are currently working in this direction.

We see many directions in which this work can be continued. First, it would be interesting to consider the large connectivity limit for each values of $q$ using the replica method, as was done for 3-satisfiability in [29]. It would also be of interest to study the dynamics along the line of [25]. Monte-Carlo simulations of these models (or exact ground state enumerations) would also be valuable to cross-check the cavity results and to study finite-size effects. Finally, the disordered version of our model is a good candidate for a glass former in finite dimension.

\footnotetext{
${ }^{2}$ Note that $T_{\text {local }}=0$ in the fully connected p-spin, the RS transition being first order, and consistently no type I instability is found.
}

$$
* * *
$$

This work has been partially supported by EVERGROW (EU consortium FP6 IST). We thank T. Jörg for a critical reading of the manuscript.

\section{REFERENCES}

[1] Sherrington D., Kirkpatrick S., Phys. Rev. Lett. 35, 1792 (1975).

[2] Parisi G., J. Phys. A13 L115-L121, (1980). Mézard M., Parisi G., Virasoro M.A., Spin Glass Theory and Beyond (World Scientific, Singapore, 1987).

[3] Talagrand M., Annals of Math. 163, no 1, 221-263 (2006).

[4] Gross D.J., Mézard M., Nuclear Physics B, 240, 4, 431452 (1984). Derrida B., Phys. Rev. Lett. 35, 1792 (1975).

[5] Gross D.J., Kanter I., Sompolinsky H., Phys. Rev. Lett. 55, 304 (1985). Kanter I., Sompolinsky H. 1987 J. Phys. A $20 \mathrm{~L} 673$.

[6] Kirkpatrick T., Thirumalai D., Phys. Rev. Lett. 58, 2091 (1987). Kirkpatrick T., Thirumalai D., Wolynes P., Phys. Rev. A 40, 1045 (1989). Kirkpatrick T., Wolynes P., Phys. Rev. A 35, 3072 (1987).

[7] Biroli G., Mézard M., Phys. Rev. Lett. 88, 025501 (2001). Rivoire O., Biroli G., Martin O.C., Mézard M., Eur. Phys. J. B 37, 55-78 (2004).

[8] Viana L., Bray A.J., J. Phys. C 18, 3037 (1985). Thouless D.J., Phys. Rev. Lett. 56, 1082 - 1085 (1986).

[9] Mézard M., Parisi G., Eur. Phys. J. B 20, 217 (2001).

[10] Mézard M., Parisi G., J. Stat. Phys 1111 (2003).

[11] Mézard M., Parisi G., Zecchina R., Science 297, 812 (2002). Mézard M., Zecchina R., Phys. Rev. E 66, 056126 (2002).

[12] Mulet R., Pagnani A., Weigt M., Zecchina R., Phys. Rev. Lett. 89, 268701 (2002). Braunstein A., Mulet R., Pagnani A., Weigt M., Zecchina R., Phys. Rev. E 68, 036702 (2003).

[13] van Mourik J., Saad D., Phys. Rev. E 66, 056120 (2002).

[14] Krząkała F., Pagnani A., Weigt M., Phys. Rev. E 70, 046705 (2004).

[15] Mézard M., Palassini M., Rivoire O., Phys. Rev. Lett. 95, 200202 (2005).

[16] Mézard M., Montanari A., J. Stat. Phys. 124, 1317 (2006).

[17] Krząkała F., Montanari A., Ricci-Tersenghi F., Semerjian G., Zdeborová L., Proc. Natl. Acad. Sci. 104, 10318 (2007).

[18] Zdeborová L., Krząkała F., Phys. Rev. E 76, 031131 (2007).

[19] Elderfield D., Sherington D., J. Phys. C 16, L497 (1983).

[20] Nishimori H., Stephen M.J., Phys. Rev. B 27, 5644 - 5652 (1983); Marinari E., Mossa S. and Parisi G., Phys. Rev. $B$ 59, 8401-8404 (1999).

[21] Montanari A., Semerjian G., J. Stat. Phys. 124, 103 (2006).

[22] Monasson R., Phys. Rev. Lett. 75, 2847 (1995).

[23] Montanari A., Parisi G., Ricci-Tersenghi F., J. Phys. A 37, 2073 (2004); Mertens S., Mézard M., Zecchina R., Rand. Struct. and Algo. 28, 340 (2006).

[24] Gardner E., Nucl. Phys. B 297, [FS14] 74 (1985).

[25] Montanari A., Ricci-Tersenghi F., Eur. Phys. J. B 33, 339 (2003). 
[26] Krząkała F., Martin O.C., Europhys. Lett. 6, 749 (2001).

[27] Franz S., Mézard M., Ricci-Tersenghi F., Weigt M., Zecchina R., Europhys. Lett. 55, 465 (2001).

[28] Mézard M., Ricci-Tersenghi F., Zecchina R., J. Stat. Phys. 111, 505 (2002).

[29] Leuzzi L., Parisi G., J Stat Phys 103679 (2001); Crisanti A., Leuzzi L., Parisi G., J. Phys. A 35, 481 (2002). 